\title{
Four-Dimensional (4D) Printing: A New Evolution in Computed Tomography-Guided Stereolithographic Modeling. Principles and Application
}

\author{
Michael P. Chae, MBBS, BMedSc ${ }^{1,2,3}$ David J. Hunter-Smith, MBBS, MPH, FRACS, FACS ${ }^{1,2,3}$ \\ Inoka De-Silva, MBBS ${ }^{2}$ Stephen Tham, MBBS, FRACS ${ }^{2}$ Robert T. Spychal, MBChB, MD, FRACS ${ }^{1,2,3}$ \\ Warren Matthew Rozen, MBBS, BMedSc, MD, PhD ${ }^{1,2,3}$
}

${ }^{1}$ Department of Plastic and Reconstructive Surgery, Frankston
Hospital, Victoria, Australia
${ }^{2}$ Department of Surgery, Monash University, Victoria, Australia
${ }^{3}$ Monash University Plastic and Reconstructive Surgery Research
Group, Peninsula Campus, Victoria, Australia

J Reconstr Microsurg 2015;31:458-463.

\begin{abstract}
Address for correspondence Warren Matthew Rozen, MBBS, BMedSc, MD, PhD, Department of Plastic and Reconstructive Surgery, Frankston Hospital, Peninsula Health, 2 Hastings Road, Frankston Victoria 3199 Australia (e-mail: warrenrozen@hotmail.com).
\end{abstract}

\begin{abstract}
Keywords

- 3D printing

- reconstructive surgery

- template

- 4D

- 3D model

- dimensional

Background Over the last decade, image-guided production of three-dimensional (3D) haptic biomodels, or rapid prototyping (RP), has transformed the way surgeons conduct preoperative planning. In contrast to earlier RP techniques such as stereolithography, 3D printing has introduced fast, affordable office-based manufacturing. We introduce the concept of $4 \mathrm{D}$ printing for the first time by introducing time as the fourth dimension to 3D printing.

Methods The bones of the thumb ray are 3D printed during various movements to demonstrate four-dimensional (4D) printing. Principles and validation studies are presented here.

Results 4D computed tomography was performed using "single volume acquisition" technology to reduce the exposure to radiation. Three representative scans of each thumb movement (i.e., abduction, opposition, and key pinch) were selected and then models were fabricated using a 3D printer. For validation, the angle between the first and the second metacarpals from the 4D imaging data and the 4D-printed model was recorded and compared.

Conclusion We demonstrate how 4D printing accurately depicts the transition in the position of metacarpals during thumb movement. With a fourth dimension of time, 4D printing delivers complex spatiotemporal anatomical details effortlessly and may substantially improve preoperative planning.
\end{abstract}

Modern imaging techniques have enabled accurate preoperative planning in plastic and reconstructive surgery, ${ }^{1}$ with advances translated to superior clinical outcomes and reduced operative length. In perforator flap surgery for breast reconstruction, preoperative computed tomography angiography (CTA) reliably, maps the vascular supply enabling the selection of donor site, flap, and the perforator. However, the interpretation of CTA scan data displayed on a two- received

October 27, 2014

accepted after revision

February 15, 2015

published online

April 13, 2015
Copyright $\odot 2015$ by Thieme Medical

Publishers, Inc., 333 Seventh Avenue, New York, NY 10001, USA. Tel: +1(212) 584-4662.
DOI http://dx.doi.org/ 10.1055/s-0035-1549006. ISSN 0743-684X. 
dimensional (2D) computer screen and correlating it to intraoperative findings has been relatively difficult. ${ }^{2}$ To this effect, the introduction of three-dimensional (3D) haptic biomodeling of rapid prototyping (RP) has been useful.

RP describes the process of fabricating a 3D physical model from a computer-aided design and stereolithography is the earliest RP technique adapted for medical application. ${ }^{3} \mathrm{~A}$ surgeon can interact with the haptic models in a "handson" fashion during preoperative planning and additionally use them for intraoperative guidance. Tactile feedback from the biomodels, in addition to their ability to accurately represent anatomical details, enables them to provide superior spatial information than a 3D visualization on a computer screen.

Stereolithographic biomodels derived from computed tomography (CT) scans have been reported to accurately map the bony and vascular anatomy and is considered a "gold standard" in medical RP. ${ }^{2,4}$ For example, fine arterial branches that can be missed on a 2D CT scan by a radiologist or a surgeon can be detected in a 3D-reconstructed image and then physically represented in a model. ${ }^{2}$ However, stereolithography is labor-intensive, relatively slow, and expensive. ${ }^{5}$ In contrast, the development of 3D printing has provided a more convenient, faster, and more affordable, alternative RP technology.

A 3D printer extrudes melted thermoplastic material and deposits it in a layer-by-layer fashion, creating an anatomically precise final product that can be handled immediately. ${ }^{3}$ This technology has shown both practical and clinical benefits in multiple surgical disciplines, including plastic surgery where 3D-printed haptic models have been utilized for volumetric analysis, ${ }^{6}$ surgical trainee education, ${ }^{7}$ and fabricating individualized prosthesis. Recent advancements in technology have allowed the incorporation of time, the fourth dimension, to medical imaging.

Four-dimensional (4D) CT is a recent imaging technology, where an oversampled 3D CT scan data are 3D-reconstructed retrospectively according to the patient's respiratory motion. This technique reduces motion artifacts, enabling precise radiotherapy delivery to lung tumors, breast cancers, and renal tumors. Its medical application has extended to include identifying parathyroid adenomas and performing functional and morphological assessment of mechanical heart valves. In plastic surgery, $4 \mathrm{D}$ CT has been reported mostly for assessing perforator vascular dynamics. ${ }^{8-10}$ We herein describe $4 \mathrm{D}$ printing/4D modeling, where time is represented by multiple haptic models depicting the movement of metacarpals during thumb movement.

\section{Methods}

To illustrate $4 \mathrm{D}$ printing, we performed $4 \mathrm{D} \mathrm{CT}$ on the hand of a young female patient during thumb abduction, opposition, and key pinch. Haptic models of the bones were fabricated using a 3D printer and compared with their subsequent 4D CT images for accuracy. Institutional ethics approval was obtained (Peninsula Health Human Research and Ethics Committee) and the patient gave verbal and written informed consent. Measurements of the staged 3D prints were performed to validate the concept of $4 \mathrm{D}$ printing (changes in 3D prints over time).

\section{Results}

\section{Scan Acquisition}

4D CT scanning was performed using a "single volume acquisition" technique previously described for perforator imaging. ${ }^{8}$ A 320 multidetector row CT scanner (Aquilion One; Toshiba America Medical Systems, Tustin, CA) was used, with slice collimation of $0.5 \mathrm{~mm}$. A $16 \mathrm{~cm}$ craniocaudal coverage meant that no concurrent table movement was required. Exposure parameters were 100 to $135 \mathrm{kVp}$ and effective tube current 400 to $580 \mathrm{~mA}$ depending on the patient's body mass index. The patient was instructed to perform three thumb movements and each movement was individually scanned.

\section{D Printing}

3D printing utilized the digital imaging and communications in medicine files of 4D CT uploaded using Osirix software (Pixmeo, Geneva, Switzerland). ${ }^{6}$ Three scans that most accurately represented transition in the metacarpals were selected for 3D printing. "Scissor" function was used to remove anatomical parts that obstructed view. Surface-rendered 3D images ( - Fig. 1A), as well as volume-rendered images ( - Fig. 1B) were produced, with both able to be manipulated with software during analysis. They were exported as standard tessellation language files and uploaded to Cubify software (3D Systems, Rock Hill, SC) for 3D printing on a Cube 2 printer (3D Systems) (-Fig. 1C).

\section{Validation of 4D Printing}

The accuracy of the $4 \mathrm{D}$ printing technique was evaluated by calculating the change in the angle of the carpometacarpal joint within models (-Figs. 2 and 3), utilizing a method previously reported for measuring thumb opposition. ${ }^{11,12}$ In short, the authors described two angles, "abduction" angle formed by the angle between the first and second metacarpals in the palmar plane and "flexion" angle formed by the plane bounded by the palmar plane and the first metacarpal. The angles were either measured directly from the 4D CT data using the "angle" function in the Osirix software or manually using a goniometer placed on the haptic models. For standardization, the middle of the trapezium was used as a fulcrum and arms of the angle were extended up to the middle of the most distal aspect of the first or second metacarpal. In the $4 \mathrm{D} \mathrm{CT}$, the coronal view was used to identify the "abduction" angle and similarly, the sagittal view for the "flexion" angle. Where the first metacarpal "crosses" the second metacarpal during thumb opposition, the angle was indicated with a negative value.

In general, the goniometer tended to overestimate the "angles" ( - Fig. 4). During thumb abduction, the "abduction" angle remained relatively static while the "flexion" angle gradually increased. This is explained by the fact that thumb abducts superiorly from the palmar plane and does not deviate in radial or ulnar direction. Similarly, during 

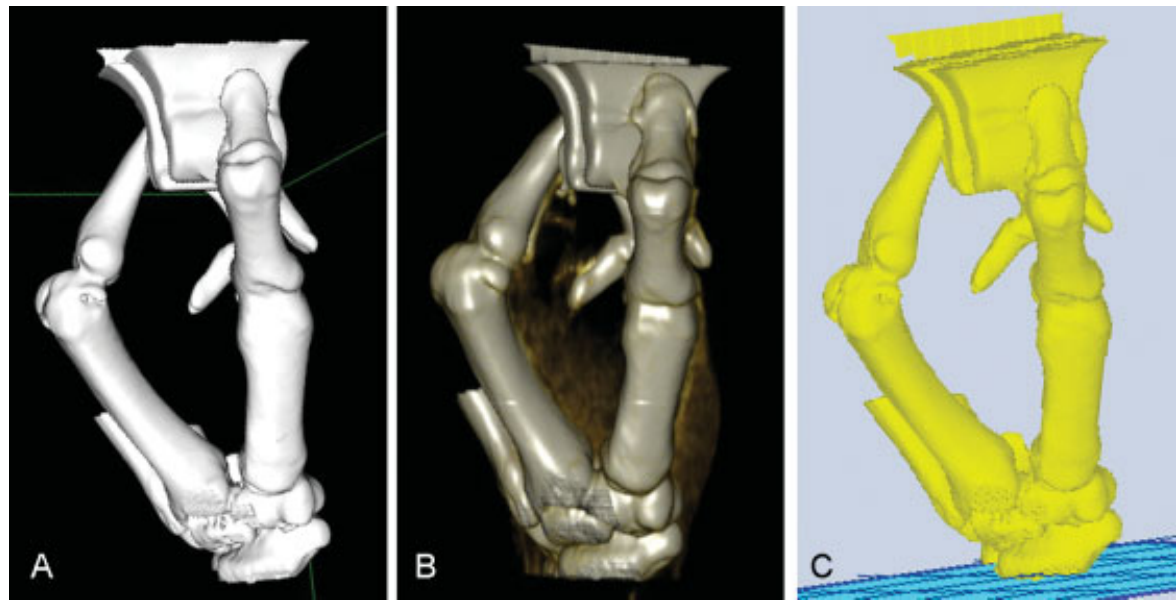

Fig. 1 CT data of the hand during key pinch is 3D reconstructed using (A) surface rendering and (B) volume rendering function in Osirix (Pixmeo, Geneva, Switzerland). (C) The 3D image is exported on to Cubify (3D Systems, Rock Hill, SC) to be rendered suitable for 3D printing. 3D, threedimensional; CT, computed tomography.

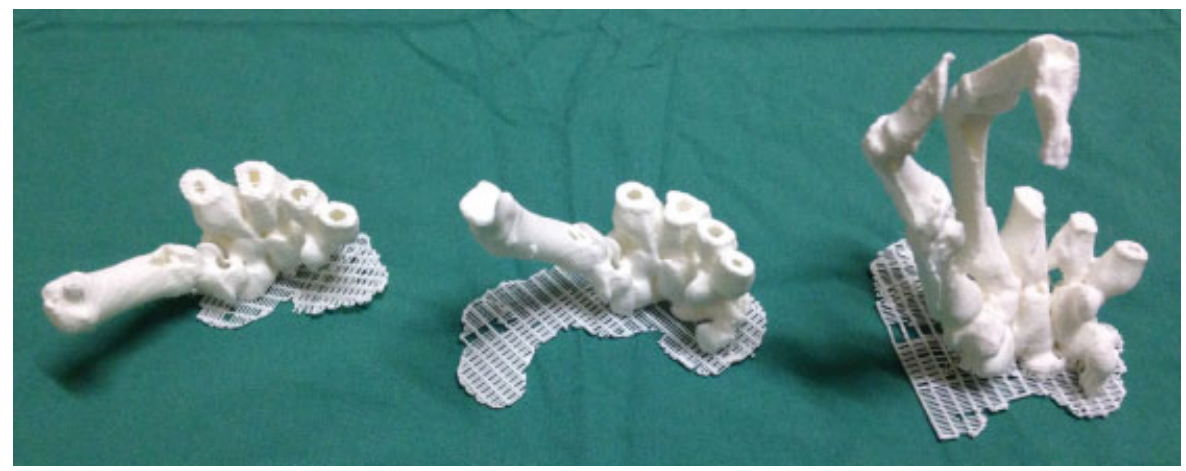

Fig. 2 3D-printed haptic models representing carpal and metacarpal bones during various hand movements: abduction (left), opposition (center), and key pinch (right). 3D, three-dimensional.

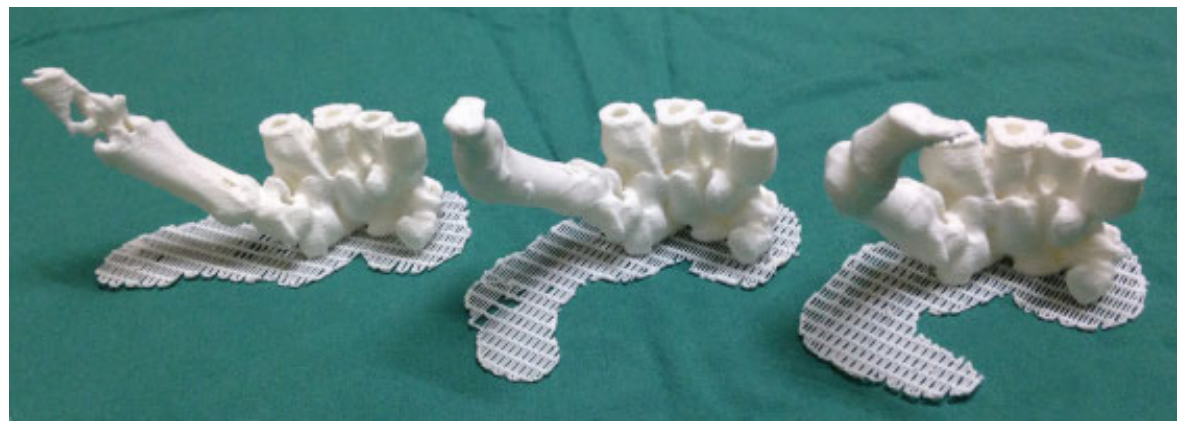

Fig. 3 4D-printing demonstrating the transition of carpal and metacarpal bones during opposition (from left to right). 4D, four-dimensional.

opposition, the thumb transitions superiorly as evidenced by increasing "flexion" angle, but additionally the "abduction" signal decreases as the thumb reaches across the palm. During key pinch, the thumb remains stationary in "flexion" angle, but the "abduction" angle decreases as the first metacarpal presses the paper clip toward the second metacarpal and subsequently, increases.

Additionally, we investigated the relationship between the base of the first metacarpal and the trapezium further in thumb opposition (-Tables 1 and 2). First, the distal articular surface of the trapezium and the part of it articulating with the base of the first metacarpal were measured using a flexible ruler. We found that as the thumb opposes, the portion of the surface of trapezium articulating with the first metacarpal increased in the ulnar direction (i.e., 53.85$84.62 \%)$. Furthermore, rotation of the first metacarpal on trapezium was detected using the angulation between the dorsal articular surface of the base of the first metacarpal and the ridge of trapezium between the first and second metacarpals. Decrease in the angle (i.e., 50-5 degrees) confirmed the rotation of the first metacarpal in the ulnar-palmar direction during thumb opposition. Moreover, we calculated 


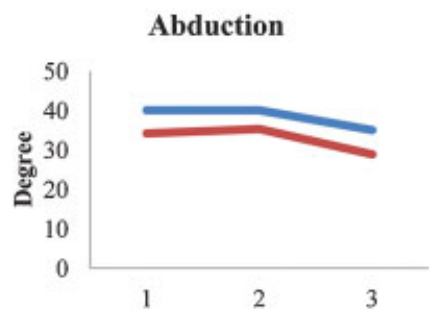

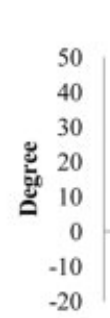

Opposition

"Abduction" Angle

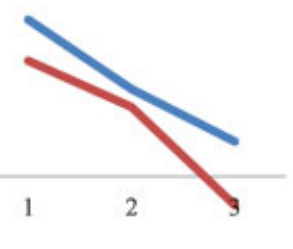

Key Pinch

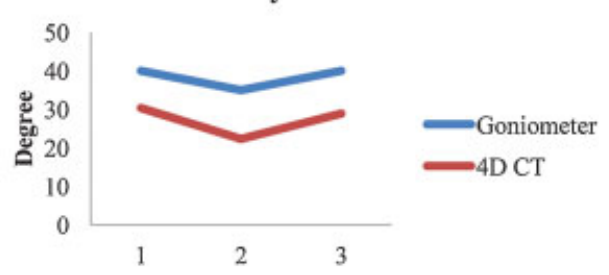

"Flexion" Angle

Abduction

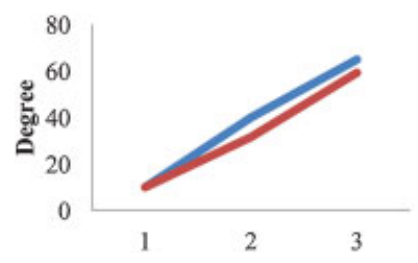

Opposition

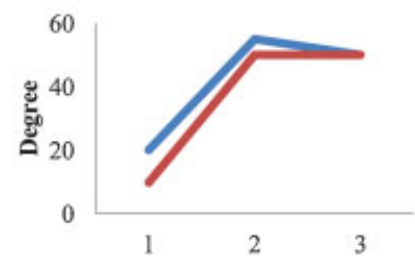

Key Pinch

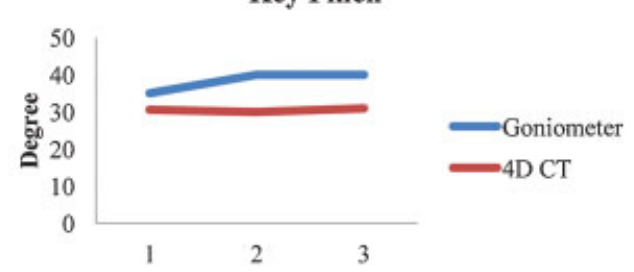

Fig. 4 Summary of graphs demonstrating the "abduction" angle (first row) and the "flexion" angle (second row) measured using a goniometer or directly from the 4D CT data. 4D, four-dimensional; $C T$, computed tomography.

Table 1 Summary of angles calculated between the first and the second metacarpal bones during various thumb movements over time (4D analysis)

\begin{tabular}{|c|c|c|c|c|c|}
\hline \multirow[t]{2}{*}{ Thumb movement } & \multirow[t]{2}{*}{ Stage } & \multicolumn{2}{|l|}{ 4D CT } & \multicolumn{2}{|l|}{ Goniometer } \\
\hline & & $\begin{array}{l}\text { Abduction angle } \\
\text { (degrees) }\end{array}$ & $\begin{array}{l}\text { Flexion angle } \\
\text { (degrees) }\end{array}$ & $\begin{array}{l}\text { Abduction angle } \\
\text { (degrees) }\end{array}$ & Flexion angle (degrees) \\
\hline \multirow[t]{3}{*}{ Abduction } & 1 & 34.15 & 10.00 & 40 & 10 \\
\hline & 2 & 35.29 & 31.56 & 40 & 40 \\
\hline & 3 & 28.84 & 59.25 & 35 & 65 \\
\hline \multirow[t]{3}{*}{ Opposition } & 1 & 33.18 & 9.82 & 45 & 20 \\
\hline & 2 & 19.90 & 50.00 & 25 & 55 \\
\hline & 3 & -8.28 & 50.07 & 10 & 50 \\
\hline \multirow[t]{3}{*}{ Key Pinch } & 1 & 30.31 & 30.60 & 40 & 35 \\
\hline & 2 & 22.33 & 30.00 & 45 & 40 \\
\hline & 3 & 28.88 & 30.95 & 40 & 40 \\
\hline
\end{tabular}

Abbreviations: 4D, four-dimensional; CT, computed tomography.

Table 2 Quantifying the relationship between the articulating surfaces of the base of first metacarpal and trapezium over time (4D analysis)

\begin{tabular}{|l|l|l|l|l|}
\hline Thumb movement & Stage & $\begin{array}{l}\text { Articular surface of } \\
\text { trapezium covered by the } \\
\text { first metacarpal (\%) }\end{array}$ & $\begin{array}{l}\text { Angle between articular surfaces of } \\
\text { trapezium and the } \\
\text { first metacarpal (degrees) }\end{array}$ & $\begin{array}{l}\text { Distance between } \\
\text { trapezium and pisiform } \\
\text { (mm) }\end{array}$ \\
\hline \multirow{2}{*}{ Opposition } & 1 & 53.85 & 50 & 28 \\
\cline { 2 - 5 } & 2 & 69.23 & 10 & 27 \\
\cline { 2 - 5 } & 3 & 84.62 & 5 & 27 \\
\hline
\end{tabular}

Abbreviation: 4D, four-dimensional.

the distance between the tubercle of trapezium and pisiform to quantify the movement of trapezium, selected since previous reports showed that trapezium moves as a single entity with trapezoid, capitate, and hamate. ${ }^{13,14}$ Although it was more obvious to the naked eye, there was only a small reduction in the distance measured (28-27 mm). This may 
be due to using a ruler that is accurate to only $1 \mathrm{~mm}$, and hence, any difference less than $1 \mathrm{~mm}$ may have been approximated up or down to the nearest millimeter.

\section{Discussion}

This original description of the technique of $4 \mathrm{D}$ printing has the potential to substantially change the way surgeons approach diagnostic and treatment planning. 2D imaging provides only a single overlapping images of a vast amount of volumetric data, as in a plain X-ray. 3D imaging provides multiple sequential images for analysis, as in CT. However, often the 3D data are still presented in only 2D format, as in the axial slice of a CT scan. 3D volumetric modeling provides the visual reformat of a 3D dataset, but again this is limited by representation of the data on a $2 \mathrm{D}$ computer monitor. In reconstructive surgery, and indeed other surgical specialties, $2 \mathrm{D}$ imaging such as plain radiography and $\mathrm{CT}$, have become integral components of preoperative workup. ${ }^{1}$ During perforator flap surgery, CTA has become a gold standard imaging modality in the evaluation of the donor site and the recipient site for the selection of an appropriate donor site and vasculature. ${ }^{1}$ Accurate anatomical details are demonstrated that can improve clinical outcomes, such as reduced donor-site morbidity, shortened operative length, and decreased overall operative complications. However, the spatiotemporal information from 2D imaging is difficult to appreciate intuitively and comparing it to clinical findings intraoperatively has also been challenging. 3D haptic models eliminate these difficulties by providing spatial anatomical information in a tactile manner that is more instinctive. 3D prints and models are thus truly presented in a 3D manner.

RP has been utilized in industrial design for decades before it was adopted for medical application in the last decade. 3D prototypes built from 3D imaging, such as CT or magnetic resonance imaging, have enabled surgeons from various disciplines to preoperatively plan, simulate procedures, and predict outcome. ${ }^{5}$ In plastic and reconstructive surgery, they have been useful for the mapping of soft tissue defects, breasts, and vasculature, and can be sterilized for intraoperative use. Biomodels also facilitate the education of surgical trainees, the potential improvement in clinicianpatient communication, and manufacturing of customized prosthesis.

Definitions of the range of techniques for 3D printing are important, as all recent and future advances in this field (which are likely to occur rapidly in this evolving domain), are based on these techniques. Stereolithography is the earliest 3D printing technology, in which a layer of liquid photopolymer or epoxy resin in a vat is cured by a low-power ultraviolet (UV) laser. Excess raw materials and the supporting structures must be manually removed from the final product and cured in a UV chamber. Multijet modeling, also known as Multijet printing (3D Systems) or PolyJet technology (Stratasys, Edina, MN), is akin to stereolithography; however, the liquid photopolymer is immediately cured by the UV light, preventing time-consuming postprocessing in the UV chamber and the prototypes are built with gel-like support mate- rials that are readily dissolvable in water. Selective laser sintering is a process where powdered forms of thermoplastic, metal, glass or ceramic material, are sintered by highpower laser beams in a layer-by-layer fashion. Binder jetting, or the powder bed technique is where printer heads eject a binder material, combined with colored dye, on to a layer of powder, fusing them layer-by-layer into a plaster model. Fused deposition modeling is currently the most commonly used consumer 3D-printing technology available in which a melted filament of thermoplastic material is extruded from a nozzle moving in the $x-y$ plane and solidifies upon deposition on a build plate. After each layer, the build plate is lowered $0.1 \mathrm{~mm}$ and the process is repeated until the final product is produced.

Stereolithography is the earliest RP technique used for medical application and is considered the current "gold standard." Despite its ability to manufacture accurate templates in a large size, stereolithography requires considerable manual handling during production, is relatively slow, and remains relatively more expensive. In contrast, more novel 3D printing technology can similarly fashion anatomically accurate haptic prototypes in a faster and relatively more affordable manner. ${ }^{3,5}$ Moreover, as it is currently garnering significant interest from the general public and the researchers, 3D printing has the potential to become far more capable, widespread, and less costly.

Recently, 4D CT technique has been introduced to improve the image quality of CT compromised by motion artifacts from organs, mainly the lungs. 4D CT is performed by oversampling images at each position of interest and associating each image to its respective breathing signal during a respiratory cycle. The data are retrospectively analyzed and yields a time-lapse geometrical dataset. In reconstructive surgery, the technology has been primarily utilized for assessing the vascular territory and flow dynamics of perforators. ${ }^{9,15}$ Initial concerns regarding high radiation exposure from 4D CT has been stymied by modifying scanning protocols. We have reported a "single volume acquisition" scanning method that significantly reduces the radiation dose to $1.78 \mathrm{mSv}$ equivalent to three plain abdominal radiographs. ${ }^{16}$ However, relative contraindications to $4 \mathrm{D}$ CT still exist, including poor renal function or allergies to iodinated contrast.

We describe 4D printing where the length, width, and height constitute three dimensions and time represents a change in the position of bones to comprise the fourth dimension. This 3D printing of 4D CT scans produces haptic biomodels that demonstrate the translation of metacarpal bones during thumb movements. Although our small sample size prohibits significant statistical derivation, the data demonstrate a strong correlation of the anatomical spatiotemporal information between the $4 \mathrm{D}$-printed biomodels and the 4D CT data.

4D printing has been previously coined in architectural design by Skylar Tibbits from the self-assembly laboratory of Massachusetts Institute of Technology (MIT), ${ }^{17,18}$ where a 3D-printed object composed of multiple materials with differing chemical properties becomes "activated" upon contact with water, and the model able to contract, expand, 
fold, and adapt to the environment. This fourth dimension was defined as the embedded capability of transforming from one shape to another. ${ }^{18}$ Although this model of "selfassembly" may eventually be adapted for medical engineering and application, our proposition of using time as the fourth dimension is conceptually more intuitive and more direct implication for utility in surgical planning. The term $4 \mathrm{D}$ modeling may thus be more appropriate going forward.

From stereolithographic biomodels to 3D-printed haptic models and now 4D printing, image-guided biomodeling has evolved dramatically. In the near future, aided by increasing availability of the $4 \mathrm{D}$ CT scanners, $4 \mathrm{D}$ printing/4D modeling has the potential to become widely accessible to provide superior spatiotemporal anatomical information for surgeons to improve clinical outcomes.

Note

The content of this article has not been submitted or published elsewhere. There was no source of funding for the article. The authors declare that there is no source of financial or other support, or any financial or professional relationships, which may pose a competing interest. All authors contributed to the preparation of this article and are in agreement with the content of the article.

\section{References}

1 Rozen WM, Ashton MW. Modifying techniques in deep inferior epigastric artery perforator flap harvest with the use of preoperative imaging. ANZ J Surg 2009;79(9):598-603

2 Rozen WM, Ting JW, Baillieu C, Leong J. Stereolithographic modeling of the deep circumflex iliac artery and its vascular branching: a further advance in computed tomography-guided flap planning. Plast Reconstr Surg 2012;130(2):380e-382e

3 Chae MP, Hunter-Smith DJ, Rozen WM. Image-guided 3D-printing and haptic modeling in plastic surgery. In: Saba L, Rozen WM, Alonso-Burgos A, Ribuffo D, eds. Imaging in Plastic Surgery. London, UK: CRC Taylor and Francis Press; 2014

4 Rozen WM, Ting JW, Leung M, Wu T, Ying D, Leong J. Advancing image-guided surgery in microvascular mandibular reconstruction: combining bony and vascular imaging with computed to- mography-guided stereolithographic bone modeling. Plast Reconstr Surg 2012;130(1):227e-229e

5 Cohen A, Laviv A, Berman P, Nashef R, Abu-Tair J. Mandibular reconstruction using stereolithographic 3-dimensional printing modeling technology. Oral Surg Oral Med Oral Pathol Oral Radiol Endod 2009;108(5):661-666

6 Chae MP, Hunter-Smith DJ, Spychal RT, Rozen WM. 3D volumetric analysis for planning breast reconstructive surgery. Breast Cancer Res Treat 2014;146(2):457-460

7 Gerstle TL, Ibrahim AM, Kim PS, Lee BT, Lin SJ. A plastic surgery application in evolution: three-dimensional printing. Plast Reconstr Surg 2014;133(2):446-451

8 Rozen WM, Chubb D, Crossett M, Ashton MW. The future in perforator flap imaging: a new technique to substantially reduce radiation dose with computed tomographic angiography. Plast Reconstr Surg 2010;126(2):98e-100e

9 Nie JY, Lu LJ, Gong X, Li Q Nie JJ. Delineating the vascular territory (perforasome) of a perforator in the lower extremity of the rabbit with four-dimensional computed tomographic angiography. Plast Reconstr Surg 2013;131(3):565-571

10 Colohan S, Wong C, Lakhiani C, et al. The free descending branch muscle-sparing latissimus dorsi flap: vascular anatomy and clinical applications. Plast Reconstr Surg 2012;130(6): 776e-787e

11 Kapandji AI. Clinical evaluation of the thumb's opposition. J Hand Ther 1992;5(2):102-106

12 Kapandji A, Moatti E, Raab C. [Specific radiography of the trapezometacarpal joint and its technique (author's transl)]. Ann Chir 1980;34(9):719-726

13 Taleisnik J. The ligaments of the wrist. J Hand Surg Am 1976;1(2): 110-118

14 Sandow MJ, Fisher TJ, Howard CQ, Papas S. Unifying model of carpal mechanics based on computationally derived isometric constraints and rules-based motion - the stable central column theory. J Hand Surg Eur Vol 2014;39(4):353-363

15 Saint-Cyr M, Schaverien M, Arbique G, Hatef D, Brown SA, Rohrich RJ. Three- and four-dimensional computed tomographic angiography and venography for the investigation of the vascular anatomy and perfusion of perforator flaps. Plast Reconstr Surg 2008; 121(3):772-780

16 Rozen WM, Whitaker IS, Stella DL, et al. The radiation exposure of Computed Tomographic Angiography (CTA) in DIEP flap planning: low dose but high impact. J Plast Reconstr Aesthet Surg 2009; 62(12):e654-e655

17 SJET. SJET @ MIT. 4D Printing: Multi-Material Shape Change: MIT 2013. Available at: http://www.sjet.us/MIT_4D\%20PRINTING.html. Accessed on June 1, 2014

18 Tibbits S. 4D printing: multi-material shape change. Arch Design 2014;84:116-121 